\title{
Overexpression of human ortholog of mammalian enabled (hMena) is associated with the expression of mutant p53 protein in human breast cancers
}

\author{
AKIHIRO TOYODA $^{1 *}$, AYA YOKOTA $^{1,3^{*}}$, TERUYOSHI SAITO ${ }^{1 *}$, HIDETADA KAWANA $^{1,4}$, \\ MORIHIRO HIGASHI ${ }^{1,5}$, YOSHIO SUZUKI ${ }^{3}$, TOMOAKI TANAKA ${ }^{2}$, \\ MOTOO KITAGAWA $^{1}$ and KENICHI HARIGAYA ${ }^{1}$
}

\author{
Departments of ${ }^{1}$ Molecular and Tumor Pathology (E4), ${ }^{2}$ Clinical Cell Biology (F5), Chiba University \\ Graduate School of Medicine, 1-8-1 Inohana, Chuo-ku, Chiba 260-8670; ${ }^{3}$ Surgical Pathology Section, \\ Asahi Central Hospital, I-1326 Asahi, Chiba 289-2511; ${ }^{4}$ Division of Surgical Pathology, Chiba Cancer Center, \\ 666-2 Nitona-Cho, Chuo-Ku, Chiba 260-8717; ${ }^{5}$ Department of Pathology, Saitama Medical Center, \\ Saitama Medical University, 1981 Kamoda, Kawagoe-shi, Saitama 350-8550, Japan
}

Received July 15,2010; Accepted September 16, 2010

DOI: 10.3892/ijo_00000827

\begin{abstract}
The human ortholog of mammalian enabled (hMena), a member of the enabled/vasodilator-stimulated phosphoprotein (Ena/VASP) family, is an actin regulatory protein involved in the regulation of cell motility. Increasing evidence suggests that hMena overexpression is involved in human cancers, but the upstream events that influence the expression of hMena remain to be elucidated. In this study, we performed immunohistochemical analysis of the expression of hMena protein in paraffin-embedded archival tissues of infiltrating ductal carcinomas (IDCs) obtained from 52 cases. We found that elevated hMena expression is associated with larger tumor size $(>2.5 \mathrm{~cm}, \mathrm{p}<0.01)$, HER2 expression $(\mathrm{p}<0.05), \mathrm{p} 53$ index $(\mathrm{p}<0.03)$ and Ki67 index $(\mathrm{p}<0.01)$, suggesting that hMena is a predictor of poor prognosis in IDCs. The histological characteristics of each specimen showed that hMena was overexpressed in the tumor cells at the invasive front of IDCs, indicating that hMena expression is at least partly mediated by tumor cell-matrix interactions. To explore the role of the absence of p53 function in hMena overexpression of IDCs, wild-type p53 cDNA was introduced into SW620 cells, which originally express mutant p53. In wild-type p53-transfected cells, hMena mRNA expression was decreased to $70 \%$ of the levels in mock transfected cells $(\mathrm{p}<0.01)$. In conclusion, our study indicates
\end{abstract}

Correspondence to: Dr Akihiro Toyoda, Department of Molecular and Tumor Pathology (E4), Chiba University Graduate School of Medicine, 1-8-1 Inohana, Chuo-ku, Chiba 260-8670, Japan

E-mail: atoyoda@faculty.chiba-u.jp

*Contributed equally

Key words: hMena, Ena/VASP, p53, breast, infiltrating ductal carcinoma that hMena overexpression is involved in the progression of IDCs, and raises the possibility that wild-type p53 may suppress hMena expression.

\section{Introduction}

Breast cancer is one of the most common malignant diseases in Western (1) and Japanese women. In Western countries, early diagnosis through screening programs and advanced therapeutic strategies (e.g., surgical technique, chemoradiotherapy and molecular targeted therapies), have significantly reduced the mortality of breast cancer. However, in Japan, mortality due to breast cancer remains high, with a rate of 11.7 deaths per 100,000 individuals, and the mortality rate is actually increasing (2). Therefore, precise prediction of the prognosis is particularly important to improve the clinical outcomes, because more aggressive (adjuvant) treatment of high-risk patients is clearly necessary.

To predict the outcome of each patient, clinicians have evaluated various parameters, such as classical anatomical classification, age, vascular invasion, and hormone receptors. Moreover, the overexpression of some molecular markers, including uPA, PAI-1, p53, Ki67, EGFR (HER2) and cyclin E, is associated with poor prognosis (3-7). Furthermore, many studies have focused on the expression of genes involved in invasion and metastasis (8-10), because it is not the primary tumor, but rather local invasion and metastases at distant sites that are the main cause of morbidity and mortality.

The invasion or metastasis of cancer cells involves multiple steps, including dissociation from the primary tumor, invasion of surrounding stroma, and intravasation into the vascular system. During these steps, the invasive carcinoma cells acquire a migratory phenotype characterized by increased expression of several genes that regulate actin polymerization. These genes are also required for cell migration in normal, non-neoplastic cells, including embryonic morphogenesis, wound healing and immune-cell trafficking (11). Therefore, 
it is critical to analyze the expression of genes that regulate actin polymerization, to better understand the nature of cancer invasion and metastasis.

Human ortholog of mammalian enabled (hMena), a member of the Ena/vasodilator-stimulated phosphoprotein (VASP) family of proteins, is a key molecule in the regulation of actin filament dynamics, and protects actin filaments from capping proteins at their barbed end (12). Recently, it has become evident that hMena may have a role in human cancer. Indeed, hMena was reported to be overexpressed in breast cancer and colorectal cancers, and its overexpression was correlated with poor prognosis (13-15). Modugno et al reported that the expression of hMena is HER2-dependent, and that the expression of hMena and HER2 is correlated with breast carcinogenesis (14). However, the upstream regulator of hMena expression remains to be determined.

In this study, we examined the expression of hMena and other molecular markers for poor prognosis of breast cancer by immunohistochemistry, and compared these results with the clinicopathological characteristics of each case to evaluate the role of hMena expression as a novel diagnostic marker for predicting the prognosis of breast cancer. We also performed in vitro studies to identify the molecule that regulates the expression of hMena.

\section{Patients and methods}

Patients and tumor tissue. Archival specimens collected between 2005 and 2006 from 52 patients with infiltrating ductal carcinoma (IDC) of the breast were studied. All patients were surgically treated with modified radical mastectomy and axillary lymph node dissection at Asahi General Hospital (Chiba, Japan). All samples were collected with approval from the ethics committee of Asahi General Hospital. The clinicopathological characteristics of each patient are shown in Table I.

The primary tumor specimens were fixed in $10 \%$ buffered formalin and embedded in paraffin using routine tissue processing methods. The diagnosis and histological grade were confirmed by reviewing the hematoxylin/eosin (HE)stain slides prepared from paraffin blocks by two pathologists (A. Yokota and A. Toyoda).

Cell culture. The SW620 colon cancer cell line was purchased from the American Type Culture Collection (ATCC). SW620 cells were cultured in Iscove's modified Dulbecco's medium (IMDM, Invitrogen) supplemented with $10 \%$ fetal bovine serum (FBS). HEK293 cells were also purchased from the ATCC, and were cultured in Dulbecco's modified Eagle's medium (DMEM, Nissui) supplemented with 10\% FBS. All cells were maintained under a humid atmosphere with $5 \%$ $\mathrm{CO}_{2}$, at $37^{\circ} \mathrm{C}$.

Plasmid construction. The full-length hMena cDNA was amplified from HeLa cell mRNA using the following forward (5'-GGCACCATGAGTGAACAGAGTA) and reverse (5'GCTCATAAATGTAGGGGTTTGC) primers. The PCR products were cloned into pCR2.1 (Invitrogen, Carlsbad, CA, USA), and their sequences were determined. The sequences of the 14 clones corresponded to sequences for hMena that have
Table I. Clinicopathological characteristics of 52 cases of IDC.

$\mathrm{n}(\%)$

$\begin{array}{ll}\text { Stage } & \\ \text { I } & 13(25 \%) \\ \text { II } & 29(56 \%) \\ \text { III } & 10(19 \%)\end{array}$

Tumor size

$\leq 2.5 \mathrm{~cm}$

$30(58 \%)$

$>2.5 \mathrm{~cm}$

$22(42 \%)$

Lymph node status

Positive

$19(37 \%)$

Negative

$33(63 \%)$

Microscopic type

Papillotubular

Solid tubular

Scirrhous

$17(33 \%)$

HER2

0

$1+$

$18(35 \%)$

$2+$

$18(35 \%)$

$3+$

$7(13 \%)$

p53

Negative or weakly positive $(<1 \%)$

$18(35 \%)$

Intermediately positive $(1-75 \%)$

$26(50 \%)$

Strongly positive $(\geq 75 \%)$

$8(15 \%)$

Ki67

Low $(<20 \%)$

High $(\geq 20 \%)$

$30(58 \%)$

been deposited in GenBank under accession no. NM_018212. A retroviral vector, pMxs-hMena was constructed by inserting the full-length hMena cDNA into the pMXs-neo vector (Cell Biolabs Inc.). pCMV-neo-BAM-p53wt, which contains the wild-type p53 clone, was a gift from Dr Bart Vogelstein (Howard Hughes Medical Institute and the Kimmel Cancer Center, Johns Hopkins University, USA).

Antibodies. The rabbit polyclonal antibody against hMena was generated as previously described (15). Additional commercial antibodies, the anti-Ki67 monoclonal antibody (mAB) (clone MIB-1, Dako Cytomation), the anti-p53 mAB (clone DO7, Dako Cytomation) and the anti-HER2 polyclonal antibody (clone A0485, Dako Cytomation) were also used.

Immunohistochemistry. hMena, HER2, p53 and Ki67 expression was assessed by indirect immunoperoxidase staining. Sections of formalin-fixed, paraffin-embedded archival tissue 


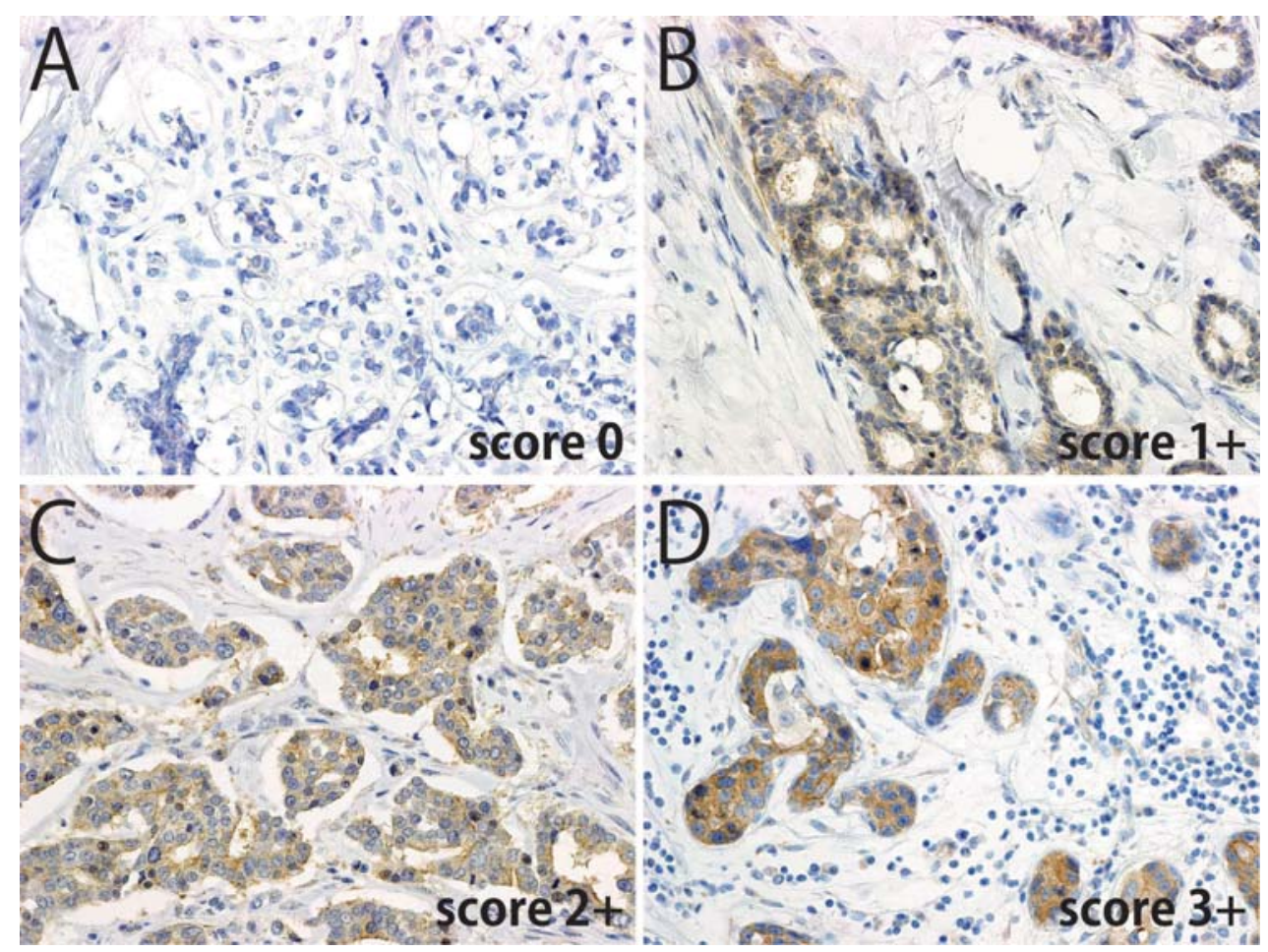

Figure 1. Representative images of hMena staining. (A) No staining (score 0). (B) Weak diffuse cytoplasmic staining in $<10 \%$ of neoplastic cells (score $1+$ ). (C) Moderate cytoplasmic staining in $10-70 \%$ of neoplastic cells (score $2+$ ). (D) Strong cytoplasmic staining in $>70 \%$ of tumor cells with or without juxtamembrane reinforcement (score $3+$ ).

(2-3 $\mu \mathrm{m}$ thick) were harvested on MDS-coated Superfrost slides (Matsunami). Sections were de-paraffinized, rehydrated, and pretreated for antigen retrieval by microwave treatment for $10 \mathrm{~min}$ in $10 \mathrm{mM}$ citrate buffer, $\mathrm{pH}$ 6.0. The sections were then incubated with anti-hMena polyclonal antibody, anti-Ki67 mAB, anti-p53 mAB or anti-HER2 polyclonal antibody for $60 \mathrm{~min}$ at room temperature. The immunoreactions were revealed using horseradish peroxidase-LSAB system (Dako Cytomation) using 3,3'-diaminobenzidine (Dako Cytomation) as the chromogenic substrate. Sections were slightly counterstained with Mayer's hematoxylin, dehydrated, and mounted for microscopic visualization. As positive controls for hMena, each staining batch included human cerebellum tissue, which shows strong immunostaining for hMena.

The intensity of hMena staining, mainly detected in the cytoplasm, was scored from 0 to $3+$ as follows: no staining, score 0 ; weak diffuse cytoplasmic staining in $<10 \%$ of neoplastic cells, score $1+;$ moderate cytoplasmic staining in $10-70 \%$, score $2+$; strong cytoplasmic staining in $>70 \%$ of the tumor cells with or without juxtamembrane reinforcement, score $3+$. The immunoreactivity of hMena was completely blocked by pre-incubation with an extract of hMena-transfected colon cancer cell line. Representative results of hMena immunostaining are shown in Fig. 1.

p53 and Ki67 expression were evaluated by the percentage of stained tumor cells. HER2 overexpression was determined in accordance with the instructions for the HercepTest (Dako) using the following categories: negative result or membrane staining in $<10 \%$ of tumor cells, score 0 ; weak and incomplete membrane staining in $>10 \%$ of tumor cells, score $1+$; weak or moderate, complete membrane staining in $>10 \%$ of tumor cells, $2+$; strong, complete membrane staining in $>10 \%$ tumor cells, score $3+$.

Gene transfer and RNA interference. Retrovirus packaging was performed by transfecting pMX-hMena and pPAM3 plasmids into HEK293 cells using the calcium phosphate transfection method, as described previously (16). After incubation for $24 \mathrm{~h}$, the medium was replaced with DMEM/10\% FBS. The cell culture medium containing viral particles was collected, filtered with a Millex $0.45-\mu \mathrm{m}$ syringe filter (Millipore), and stored at $-80^{\circ} \mathrm{C}$ until use. For infection with retroviruses, the SW620 cells were plated in 6 -well plates $\left(1 \times 10^{6}\right.$ cells/well $)$ and incubated with the virus-containing medium $(1.5 \mathrm{ml} /$ well $)$ containing Polybrene ( $4 \mu \mathrm{g} / \mathrm{ml}$; Sigma) for 1 day. The blank vector (pMXs-neo) was transferred into SW620 cells using the same method.

Motility assay. The SW620 cell line was used to investigate cell motility. hMena, p53, blank vectors, control siRNA or siRNA for hMena were retrovirally expressed or transiently transfected using Lipofectamine 2000 (Invitrogen). After incubating the cells with IMDM/ $0.5 \%$ FBS overnight, the motility assays were carried out using Boyden chambers. Boyden chambers comprise a chamber with two medium-filled compartments containing different concentrations $(0.5 \% / 0.5 \%$, $10 \% / 10 \%$ and $0.5 \% / 10 \%$ ) of FBS. The lower and upper chambers are separated by a polycarbonate membrane with $8-\mu \mathrm{m}$-sized pores. Cells were allowed to migrate for $4 \mathrm{~h}$. The membrane was fixed and stained with Mayer's hematoxylin. Cells on the upper side of the membrane were removed by cotton swabs. Cells on the lower side of the membrane were 
counted using a light microscope at magnification x200. Cells were counted in four random fields, and the results are expressed as the mean \pm standard error $(\mathrm{SE})$.

Real-time PCR. To elucidate the expression pattern of hMena mRNA in morbid cells, total RNA was extracted with TRIzol (Invitrogen) according to the manufacturer's instruction. cDNA was synthesized from $500 \mathrm{ng}$ of total RNA with the PrimeScript reverse transcript reagent (Takara) according to the manufacturer's instructions. Semi-quantitative real-time PCR analysis of hMena was then performed using a Prism 7000 (Applied Biosystems) thermal cycler with SYBR pre-mix ExTaq reagent (Takara). The primers were as follows: hMena (sense, 5'-GCTGGTGGCTCAACTGGATTC-3'; antisense, 5'-CCTGTCTAGCATCTCGCCACTG-3'), human GAPDH (sense, 5'-GCACCGTCAAGGCTGAGAAC-3', antisense, 5'-TGGTGAAGACGCCAGTGGA-3') and p53 (sense, 5'-CG TGTGAGTATTTTGGATGACAGA-3', antisense, 5'-TGTA GTGGATGGTGGTACAGTCAGA-3'). Each PCR cycle consisted of $10 \mathrm{sec}$ at $95^{\circ} \mathrm{C}$ and 40 repeats of $5 \mathrm{sec}$ at $95^{\circ} \mathrm{C}$ and $31 \mathrm{sec}$ at $60^{\circ} \mathrm{C}$ ). The relative expression level of hMena was calculated by the comparative threshold cycle $(\mathrm{Ct})$ method (17). All experiments were performed in triplicate, and the results were expressed as means $\pm \mathrm{SE}$.

Statistical analyses. Statistical analysis was performed using Microsoft Excel (Microsoft). Paired-sample t-tests, $\chi^{2}$ test, or Kruskal-Wallis test were used as appropriate. P-values $\leq 0.05$ were considered statistically significant.

\section{Results}

hMena expression is increased in breast cancer. We used indirect immunohistochemistry to evaluate the expression of hMena in paraffin-embedded breast tissue samples from patients with IDCs. The clinicopathological characteristics of the cases are summarized in Table I. In the normal breast tissue, hMena staining was found in the duct epithelia, myoepithelia, neural tissue and basal cell layer of the skin, although its expression was weak or almost undetectable, and the expression levels varied. In contrast, the expression of hMena tended to be greater in IDCs than in normal epithelia (Fig. 1). hMena staining was mainly localized to the cytoplasm, although membranous/juxtamembranous enhancement was observed in some cases. We graded the intensity of hMena staining in IDCs with a four-point grading system ( 0 to $3+)$ in accordance with the staining intensity of the tumor cells in the most central section in tumors, and scores of $1+$ or higher were regarded as positive staining of hMena. Based on this scoring system, hMena expression was detected in all IDC specimens (52 of 52, 100\%), suggesting that hMena is overexpressed in IDCs.

hMena expression is associated with larger tumor size. We next examined the correlations between various clinicopathological characteristics of the tumors and the intensity of hMena expression (staining score). As shown in Table II, larger tumor size (greatest diameter $>2.5 \mathrm{~cm}$ ) was correlated with higher hMena score $\left(\mathrm{p}=0.0305, \chi^{2}\right.$ test). In contrast, clinical stage, lymph node status and histotype were not
Table II. Associations between hMena score and clinicopathological characteristics.

\begin{tabular}{lccc}
\hline A, Clinical stage & & & \\
& 1+(weak) & 2+(moderate) & $3+$ (strong) \\
\hline I & 4 & 10 & 8 \\
II & 3 & 10 & 5 \\
III & 0 & 6 & 6 \\
\hline
\end{tabular}

$\mathrm{p}=0.36$, Kruskal-Wallis test.

(B) Tumor size

$1+($ weak $) \quad 2+($ moderate $) \quad 3+($ strong $)$

\begin{tabular}{llrr}
\hline Small $(\leq 2.5 \mathrm{~cm})$ & 8 & 16 & 6 \\
Large $(>2.5 \mathrm{~cm})$ & 0 & 9 & 13 \\
\hline
\end{tabular}

$\mathrm{p}=0.0305, \chi^{2}$ test $\left(\chi^{2}=11.58\right)$.

(C) Histotype

$1+($ weak $) \quad 2+($ moderate $) \quad 3+($ strong $)$

\begin{tabular}{llrl}
\hline Papillotubular & 2 & 13 & 9 \\
Solid-tubular & 3 & 5 & 3 \\
Scirrhous & 4 & 7 & 7 \\
\hline
\end{tabular}

$\mathrm{p}=0.58 ; \chi^{2}$ test $\left(\chi^{2}=2.84\right)$.

(D) World Health Organization histological grade $1+($ weak $) \quad 2+$ (moderate) $3+$ (strong)

\begin{tabular}{lrrr}
\hline G1 & 3 & 4 & 1 \\
G2 & 4 & 12 & 7 \\
G3 & 1 & 9 & 11 \\
\hline
\end{tabular}

$\mathrm{p}=0.06$, Kruskal-Wallis test.

(E) Lymph node status

$1+($ weak $) \quad 2+($ moderate $) \quad 3+($ strong $)$

\begin{tabular}{lrrr}
\hline Negative & 2 & 9 & 8 \\
Positive & 6 & 15 & 12 \\
\hline
\end{tabular}

$\mathrm{p}=0.75, \chi^{2}$ test $\left(\chi^{2}=0.57\right)$.

significantly associated with hMena score. This result raises the possibility that hMena is involved in tumor cell proliferation.

hMena expression tends to be high in invasive front of the tumor. On closer inspection of each specimen, we found more intensive hMena staining at the invasive front of the tumor than at the center of the tumor (Fig. 2). In particular, spots indicating strong immunoreactivity for hMena were 


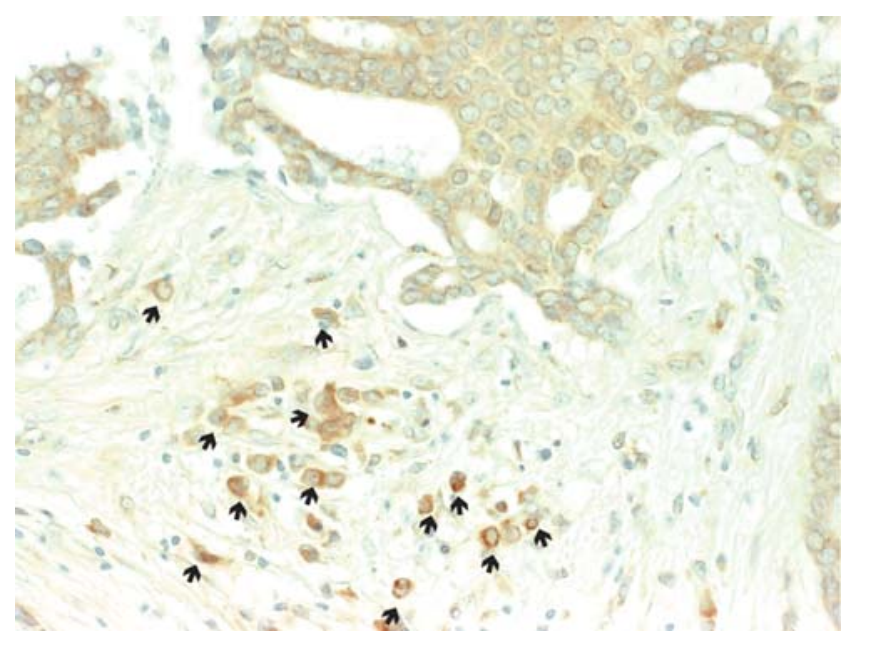

Figure 2. Strong hMena staining was observed in the focally dedifferentiated cancer cells at the invasive front of cancer (arrows).

frequently observed in the focally dedifferentiated cancer cells at the invasive front of cancer (Fig. 2). These findings indicate that hMena expression is strongly upregulated by cell-substrate interactions at the invasive front of IDCs.

hMena expression is correlated to HER2, p53 and Ki67 expression. Next, we assessed the correlations between the intensity of hMena staining and the expression of other wellknown prognostic markers to ascertain whether these markers are associated with the induction of hMena expression. As shown in Table III, the intensity of hMena expression was correlated with the expression of all three prognostic markers $(\mathrm{p}<0.01)$. These results indicate that hMena expression is a possible marker for poor prognosis in IDCs.

Knockdown of hMena down-regulated the migratory phenotype of SW620 cells. We have already reported that the overexpression of hMena enhances the migratory phenotype of HeLa-S3 cervical cancer cell line (15). Based on our recent findings, we performed a migration assay to elucidate whether
Table III. Associations between hMena score and other molecular markers of IDCs.

\begin{tabular}{lc}
\hline Parameter & P-value \\
\hline HER2 & $6.798 \times 10^{-20^{a}}$ \\
p53 & $3.963 \times 10^{-6^{a}}$ \\
Ki67 & $1.619 \times 10^{-5^{a}}$ \\
ER & 0.928 \\
PgR & 0.999 \\
\hline
\end{tabular}

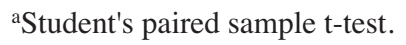

knockdown of hMena affects the migratory phenotype of cancer cells. To achieve this, we used the Boyden chamber assay, in which the upper/lower chambers contained media with the different serum concentrations (upper/lower: $0.5 \% / 0.5 \%, 0.5 \% / 10 \%$ and $10 \% / 10 \%$ ) to emulate chemotactic/ chemokinetic activities. As shown in Fig. 3, knockdown of hMena significantly down-regulated migratory phenotypes of SW620 cells $(p<0.01)$, suggesting that the expression levels of hMena can influence cancer cell migration.

Introduction of wild-type p53 cDNA reduced hMena expression and down-regulated the migratory phenotype of SW620 cells. In accordance with the immunohistochemistry findings, we postulated that the loss of p53 function may contribute to the overexpression of hMena. To test this possibility, we performed in vitro studies using the SW620 colon cancer cell line, which contains a point mutation in codon 273 ( $\mathrm{Arg} \rightarrow \mathrm{His})$ of the p53 protein (18). As shown in Fig. 4, the introduction of wild-type p53 cDNA decreased hMena mRNA levels to $70 \%$ ( $\mathrm{p}<0.01$, Student's t-test). We also carried out a Boyden chamber assay to elucidate whether p53 can influence the migratory phenotypes of SW620 cells. We found that the introduction of wild-type p53 significantly decreased the number of migratory cells in the $0.5 \% / 10 \%$ and $10 \% / 10 \%$
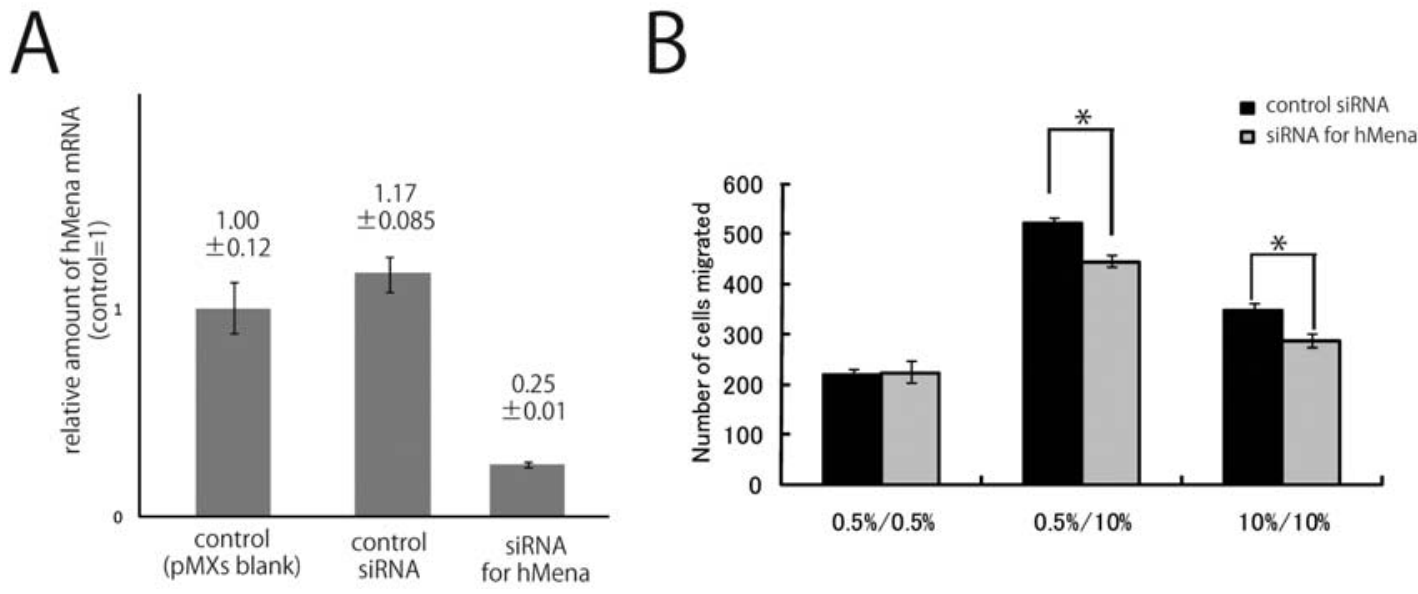

Figure 3. Knockdown of hMena by specific siRNA significantly reduced the chemotactic/chemokinetic activities of SW620 cells. ${ }^{*}$ p $<0.01$. 

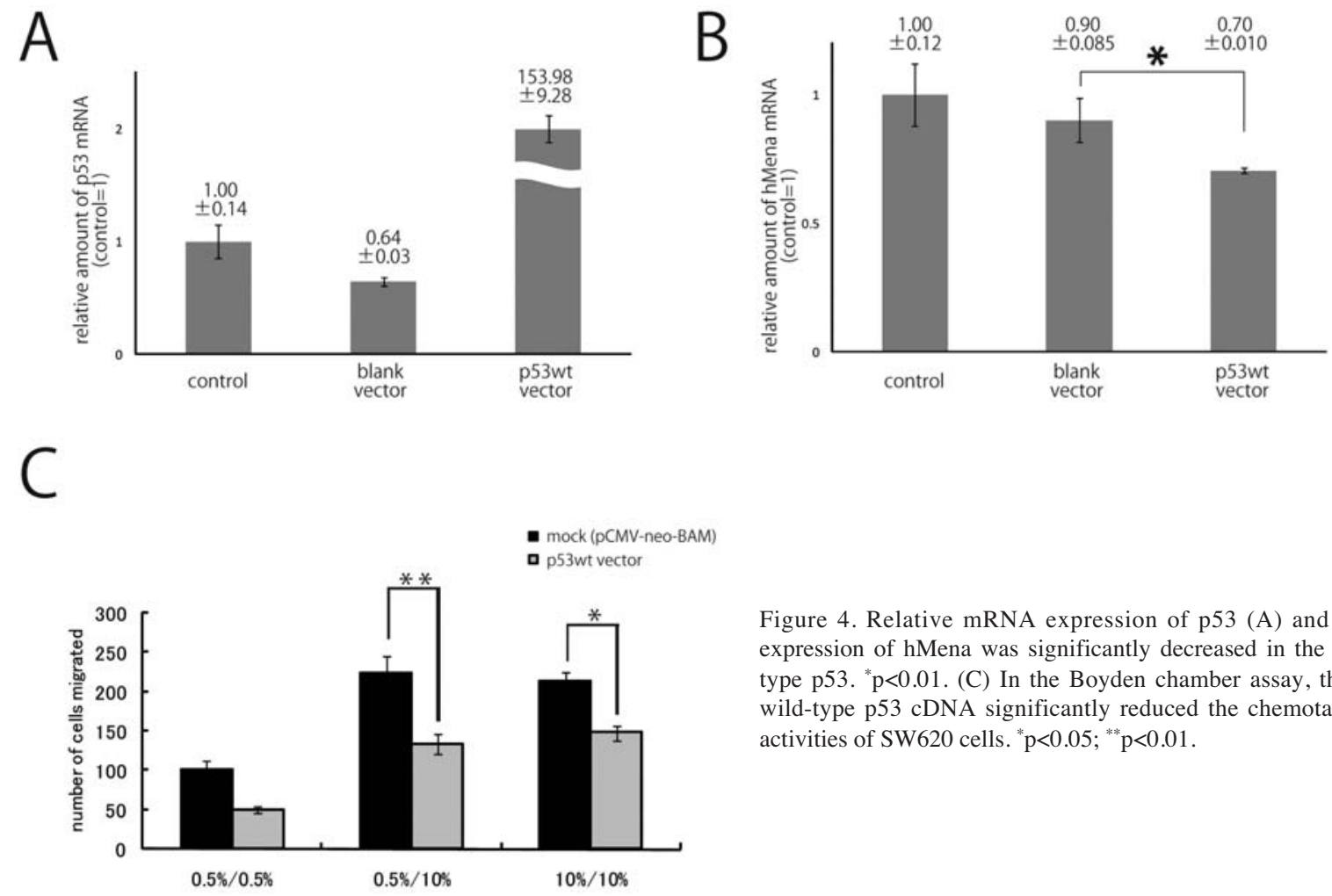

Figure 4. Relative mRNA expression of p53 (A) and hMena (B). The expression of hMena was significantly decreased in the presence of wildtype p53. ${ }^{*}<0.01$. (C) In the Boyden chamber assay, the introduction of wild-type p53 cDNA significantly reduced the chemotactic/chemokinetic activities of SW620 cells. ${ }^{*} \mathrm{p}<0.05 ;{ }^{* *} \mathrm{p}<0.01$.

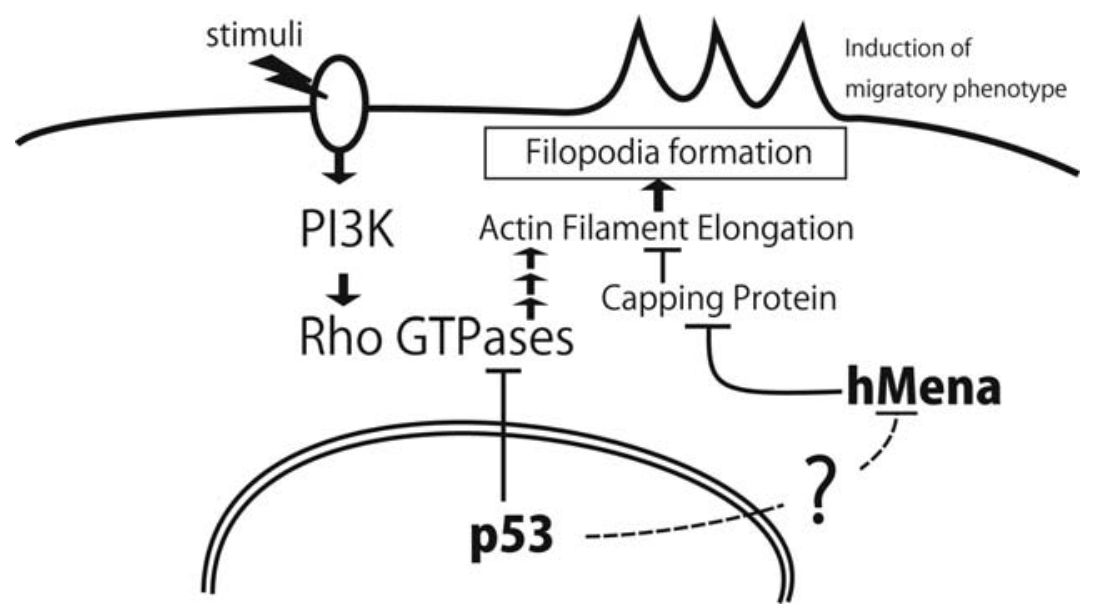

Figure 5. Schematic model showing the roles of p53 and hMena in migratory activities of invading cancer cells.

chambers. These results suggest that wild-type p53 may at least partly regulate the expression of hMena and the migratory phenotypes of cancer cells.

\section{Discussion}

The Ena/VASP family of proteins are key regulators of actin cytoskeleton dynamics involved in cell motility (12). Changes in the cellular actin network play a role in malignant transformation and tumor progression. Earlier studies have revealed enhanced expression of Ena/VASP protein in various malignant neoplasms (13-15). In this study, we showed that the expression of hMena is significantly increased in IDCs, as described in earlier reports. Although we found that the expression of
hMena was not correlated to the advanced clinical stage of tumors, hMena may be a marker for IDCs.

HER2 has an adverse effect on the prognosis of IDCs (6). We found that hMena overexpression was associated with HER2 overexpression and tumor size. Thus, our findings raise the possibility that hMena may have a role in the proliferation of IDCs in relation to HER2. Supporting our results, previous reports have demonstrated that a splice variant of hMena $\left(\mathrm{hMena}^{+11 \mathrm{a}}\right)$ is involved in breast cancer cell proliferation in response to EGF stimulation (19). Accordingly, hMena score was also positively correlated with Ki67 index. As Ki67 is a marker for proliferating cells (20) and is an established prognostic marker for breast cancer (7), hMena overexpression may be associated with the rapid-growing phenotype of IDCs. 
We also performed histological analysis of each specimen, which revealed intensive hMena staining in cell membranes at the invasive front of the tumor, suggesting that hMena overexpression is related to the invasive phenotype of cancer cells. Our recent report demonstrated that hMena may be involved in the progression of colorectal carcinoma by enhancing the migratory phenotype of cancer cells (15). In this study, we found that knockdown of hMena can inhibit the migratory phenotypes of SW620 cells. Our results suggest that hMena may augment the invasive capacity of cancer cells. Furthermore, Philippar et al reported that Mena and its isoform MenaINV can promote carcinoma cell motility and invasiveness in vivo and in vitro, and increase lung metastasis by potentiating EGF-induced membrane protrusion and increasing the matrix degradation activity of tumor cells $(21,22)$. These reports are consistent with our previous results, and we speculate that our immunohistochemical analysis partly detected the invasive isoform of hMena. Although our immunohistochemistry data are largely dependent on the visual inspection of sections by a surgical pathologist, our understanding of the nature of cancer cell invasion and the diagnosis of cancer will be enhanced if novel procedures can detect the invasive isoform of hMena in human tissues.

p53, which inhibits the cell cycle and regulates apoptosis, for example, is involved in various stages of carcinogenesis and cancer progression (23). The wild-type p53 protein normally acts in the nucleus and has several nuclear localization signals, which are necessary but not sufficient to direct it to this cellular location. Accordingly, wild-type p53 is almost undetectable. However, mutant p53 has a longer half-life and greater stability compared with wild-type p53 (24) and nuclear accumulation of mutant p53 is more easily detected. Thus, aberrant nuclear p53 expression is thought to be a marker for the aggressiveness of breast cancer (4). We found that hMena expression was significantly correlated to p53 expression, supporting the use of hMena as a marker for the aggressiveness of the IDCs.

Increasing evidence suggests that p53 is involved in cell migration and invasion (25). Guo et al reported that the loss of p53 allows Rho family GTPase-induced cell proliferation, gene transcription and migration of mouse embryonic fibroblasts $(26,27)$. In particular, TNF $\alpha$-induced cell migration is antagonistically regulated by cdc42-mediated filopodia formation and its inhibition occurs via a p53-dependent pathway (28). Because hMena contributes to filopodia formation by antagonizing the capping protein (12), it is plausible that the loss of p53 can influence the behavior of hMena in migrating cancer cells. We demonstrated that transient transfection with the wild-type p53 gene significantly decreased hMena expression and reduced the migratory phenotype of SW620 cells. Therefore, our findings suggest that the expression of hMena is related, if not directly, to the p53-dependent pathway.

In conclusion, we have demonstrated that hMena is overexpressed in IDCs and its expression is associated with other well-known prognostic markers of IDCs. In particular, we found that overexpression of hMena is closely associated with the expression of mutant p53, and that p53 may regulate the migratory phenotypes of invading cancer cells by influencing hMena expression (Fig. 5). However, the molecular mechanisms involved in the interaction between
hMena and p53 remain to be elucidated. Further studies may provide additional insight into our understanding of the nature of cancer invasion and possible therapeutic strategies for IDCs.

\section{Acknowledgements}

We are grateful to the Asahi General Hospital for surgical specimens; Dr Bart Vogelstein (Howard Hughes Medical Institute and the Kimmel Cancer Center, Johns Hopkins University) for p53 vector; T. Umemiya, K. Azuma, Dr T. Oyama and Y. Shida for help. This work was supported by a Grant-in-Aid for Scientific Research on Priority Areas 15024210 from the Ministry of Education, Culture, Sports, Science and Technology of Japan (to K. Harigaya), Grants-inAid for Scientific Research 15390122 and 22390074 from the Japan Society for the Promotion of Science (to K. Harigaya), and the funds from the Department of Molecular and Tumor Pathology, Chiba University Graduate School of Medicine.

\section{References}

1. Jemal A, Siegel R, Ward E, Hao Y, Xu J, Murray T and Thun MJ: Cancer statistics, 2008. CA Cancer J Clin 58: 71-96, 2008.

2. The Japanese Breast Cancer Society: Morbidity of breast cancers in Japanese women. Kanehara Shuppan, Tokyo, 2008.

3. Janicke F, Schmitt M, Pache L, Ulm K, Harbeck N, Hofler H and Graeff $\mathrm{H}$ : Urokinase (uPA) and its inhibitor PAI-1 are strong and independent prognostic factors in node-negative breast cancer. Breast Cancer Res Treat 24: 195-208, 1993.

4. Ostrowski JL, Sawan A, Henry L, et al: p53 expression in human breast cancer related to survival and prognostic factors: an immunohistochemical study. J Pathol 164: 75-81, 1991.

5. Porter PL, Malone KE, Heagerty PJ, et al: Expression of cell-cycle regulators $\mathrm{p} 27 \mathrm{Kip} 1$ and cyclin $\mathrm{E}$, alone and in combination, correlate with survival in young breast cancer patients. Nat Med 3: 222-225, 1997

6. Slamon DJ, Clark GM, Wong SG, Levin WJ, Ullrich A and McGuire WL: Human breast cancer: correlation of relapse and survival with amplification of the HER-2/neu oncogene. Science 235: 177-182, 1987.

7. Wiesner FG, Magener A, Fasching PA, et al: Ki-67 as a prognostic molecular marker in routine clinical use in breast cancer patients. Breast 18: 135-141, 2009.

8. Muller A, Homey B, Soto H, et al: Involvement of chemokine receptors in breast cancer metastasis. Nature 410: 50-56, 2001.

9. Van de Vijver MJ, He YD, van't Veer LJ, et al: A geneexpression signature as a predictor of survival in breast cancer. N Engl J Med 347: 1999-2009, 2002.

10. Van't Veer LJ, Dai H, van de Vijver MJ, et al: Gene expression profiling predicts clinical outcome of breast cancer. Nature 415: 530-536, 2002.

11. Friedl $\mathrm{P}$ and Wolf $\mathrm{K}$ : Tumour-cell invasion and migration: diversity and escape mechanisms. Nat Rev Cancer 3: 362-374, 2003.

12. Krause M, Dent EW, Bear JE, Loureiro JJ and Gertler FB: Ena/VASP proteins: regulators of the actin cytoskeleton and cell migration. Annu Rev Cell Dev Biol 19: 541-564, 2003.

13. Di Modugno F, Bronzi G, Scanlan MJ, et al: Human Mena protein, a serex-defined antigen overexpressed in breast cancer eliciting both humoral and $\mathrm{CD} 8^{+} \mathrm{T}$-cell immune response. Int $\mathrm{J}$ Cancer 109: 909-918, 2004

14. Di Modugno F, Mottolese M, Di Benedetto A, et al: The cytoskeleton regulatory protein hMena (ENAH) is overexpressed in human benign breast lesions with high risk of transformation and human epidermal growth factor receptor-2-positive/hormonal receptor-negative tumors. Clin Cancer Res 12: 1470-1478, 2006.

15. Toyoda A, Kawana H, Azuhata K, et al: Aberrant expression of human ortholog of mammalian enabled (hMena) in human colorectal carcinomas: implications for its role in tumor progression. Int J Oncol 34: 53-60, 2009.

16. Kingston RE, Chen CA and Rose JK: Calcium phosphate transfection. Curr Protoc Mol Biol Chapter 9: Unit 9.1, 2003. 
17. Monney L, Sabatos CA, Gaglia JL, et al: Th1-specific cell surface protein Tim-3 regulates macrophage activation and severity of an autoimmune disease. Nature 415: 536-541, 2002.

18. Rodrigues NR, Rowan A, Smith ME, Kerr IB, Bodmer WF, Gannon JV and Lane DP: p53 mutations in colorectal cancer. Proc Natl Acad Sci USA 87: 7555-7559, 1990.

19. Di Modugno F, DeMonte L, Balsamo M, et al: Molecular cloning of hMena (ENAH) and its splice variant hMena+11a: epidermal growth factor increases their expression and stimulates hMena+11a phosphorylation in breast cancer cell lines. Cancer Res 67: 2657-2665, 2007.

20. Scholzen T and Gerdes J: The Ki-67 protein: from the known and the unknown. J Cell Physiol 182: 311-322, 2000.

21. Philippar U, Roussos ET, Oser M, et al: A Mena invasion isoform potentiates EGF-induced carcinoma cell invasion and metastasis. Dev Cell 15: 813-828, 2008.

22. Goswami S, Philippar U, Sun D, et al: Identification of invasion specific splice variants of the cytoskeletal protein Mena present in mammary tumor cells during invasion in vivo. Clin Exp Metastasis 26: 153-159, 2009.
23. Coles C, Condie A, Chetty U, Steel CM, Evans HJ and Prosser J: p53 mutations in breast cancer. Cancer Res 52: 5291-5298, 1992.

24. Ginsberg D, Michael-Michalovitz D and Oren M: Induction of growth arrest by a temperature-sensitive p53 mutant is correlated with increased nuclear localization and decreased stability of the protein. Mol Cell Biol 11: 582-585, 1991.

25. Roger L, Gadea G and Roux P: Control of cell migration: a tumour suppressor function for p53? Biol Cell 98: 141-152, 2006.

26. Guo F and Zheng Y: Rho family GTPases cooperate with p53 deletion to promote primary mouse embryonic fibroblast cell invasion. Oncogene 23: 5577-5585, 2004.

27. Guo $\mathrm{F}$ and Zheng Y: Involvement of Rho family GTPases in p19Arf- and p53-mediated proliferation of primary mouse embryonic fibroblasts. Mol Cell Biol 24: 1426-1438, 2004.

28. Gadea G, Roger L, Anguille C, De Toledo M, Gire V and Roux P: TNFalpha induces sequential activation of Cdc42- and p38/p53-dependent pathways that antagonistically regulate filopodia formation. J Cell Sci 117: 6355-6364, 2004. 